\title{
Underwater radio device
}

\author{
Yurii Khlaponin', Oleksandr Selyukov² \\ Kyiv national university of construction and architecture \\ Povitroflotskiy avenue 31, Kyiv, Ukraine, 03037 \\ 1y.khlaponin@gmail.com, orcid.org/ 0000-0002-9287-0817 \\ 2selukov@3g.ua, orcid.org/ 0000-0001-7979-3434
}

Received 11.04.2020, accepted after revision 25.05.2020

https://doi.org/10.32347/uwt2020.09.1501

\begin{abstract}
Creation of a radio communication device for underwater objects for the exchange of voice messages and for the transmission of digital data. The relevance of this problem is determined by the fact that the currently existing samples of equipment for underwater radio communications are excessively powerful, dimensional and with low bandwidth. An analysis of developments in this area showed that underwater radio communications can be organized on longitudinal electromagnetic waves or by using only the magnetic component of the electromagnetic wave.

The principle of operation of the device is based on the use of only the magnetic component of the electromagnetic wave. It is known that an electromagnetic wave consists of two components: electrical and magnetic. Water is an electrically conductive medium where the skin effect acts, according to which the deeper, the weaker the signal. But this is for the electrical component. Water has weak magnetic properties, so water is not a hindrance to the magnetic component of an electromagnetic wave.

Such a device, depending on its power, can be used both for individual communication of swimmers, and for the organization of radio communication between beacons and even for communication with submarines. It is possible to isolate the magnetic component of an electromagnetic wave according to two principles: to strengthen the magnetic component or to weaken the electrical component.

The separation of the magnetic component of the electromagnetic wave is carried out in the socalled magnetic antennas, which can be built in the form of coils with a ferrite core, can be of a frame type of a certain size or in the form of a coil in a screen made of non-magnetic metal.
\end{abstract}

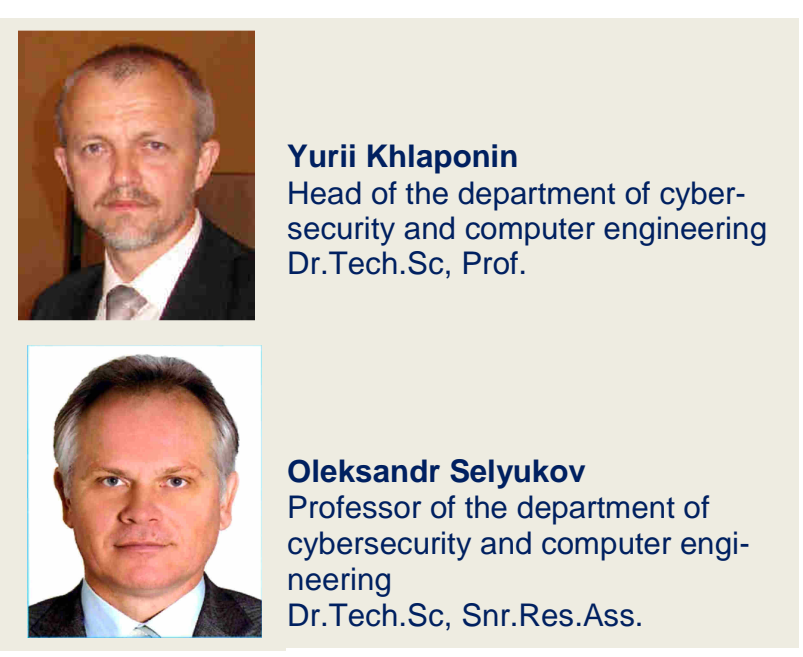

When upgrading conventional radio communication devices to the underwater version of their use, only the antenna-feeder path is subject to change, while other components of the communication channel remain unchanged. Application of such principles of radio communication organization will allow building submarine communication systems for an almost unlimited distance.

Keywords: radio communication, antenna, electromagnetic wave, electromagnetic field, magnetic component, electromotive force, submarine.

\section{INTRODUCTION}

It is known that the water of the oceans and seas has conductivity and significantly absorbs radio waves. In this environment, the radio wave almost attenuates in the thickness of the skin layer. In addition, the absorption coefficient increases with increasing frequency. 
Therefore, long and ultra-long waves are commonly used to organize underwater radio communication, and optical [1] and acoustic $[21,22]$ waves can also be used. Specific features of the aquatic environment for the propagation of electromagnetic waves [3] are bias currents are much smaller compared to conduction currents, at the skin-layer distance the resistive and inductive properties of the medium are comparable, and capacitive are not affected, there is no wave zone: at any distance from the radiation source the pointing point is much less than Joule losses, impossible formation of narrow radiation patterns, etc. Therefore, to organize long-distance communication requires powerful equipment with antennas [6], the length of which is several kilometers, and this connection is quite slow, the bandwidth of such a communication channel reaches hundreds of bits per second. Obviously, other principles, one of which is suggested in this article, need to be used to organize a high-speed communication channel and over long distances underwater.

\section{ANALYSIS OF RECENT RESEARCH AND PUBLICATIONS}

The history of communication with submarines [1] defines the basic principles that have guided the last 100 years. Long and ultra-long waves for long-distance communication (kilometers) are commonly used for underwater radio communication, and optical and acoustic waves can be used for short-distance communication (meters). According to the results of research of underground and underwater radio communication [3], the peculiarities of the aquatic environment for the propagation of the electromagnetic wave are clarified and it is determined that it is not effective to use Hertz radio waves in the aquatic environment. The article [4] discusses the issue of still insufficiently studied radiation of a rotating magnet, which can penetrate through the aqueous medium. Article [6] states that the existing system of communication with submarines involves high-power equipment, which also has large dimensions for almost submarines. Such equipment may not be used for individual communication of scuba divers. In [9] the connection between the longitudinal electromagnetic wave and the known system of Maxwell's equations is proved, where the vector of the longitudinal wave component of the electric field strength is found as an exact solution of Maxwell's standard equation with a specific case of gradient type of electric current and charge density. That is, it is possible to use longitudinal electromagnetic waves for underwater radio communication, which propagate under water almost without restrictions.

\section{THE AIM OF THE STUDY}

The urgency of this problem is determined by the fact that the current models of underwater radio equipment are excessively powerful, large and with low bandwidth. The results of the analysis are: alternative types of underwater radio communication are longitudinal electromagnetic waves and radio communication using the magnetic component of the electromagnetic wave. The purpose of the study, which is conducted in this article, is to determine the design features of equipment for the organization of radio communication using the magnetic component of the electromagnetic wave. The tasks that will be solved: to determine the principles of separation of the magnetic component from the electromagnetic wave and to determine the features of the construction of the communication channel for underwater radio devices.

\section{RESULTS OF THE RESEARCH}

Of course, the electromagnetic wave consists of two components: electric and magnetic. Water is a conducive medium where the skin effect acts, which is deeper, the weaker. But this is for the electrical component. For the magnetic component, water is not an obstacle. It is possible to distinguish the magnetic component of an electromagnetic wave according to two principles:

- amplify the magnetic component (the socalled magnetic antenna);

- weaken the electrical component (socalled HZ-antenna). 
The type of antennas that respond to the magnetic component of the electromagnetic field (EMF) has been widely used in any industry due to its small size and transceiver properties. The main advantage of magnetic antennas - immunity to electrical interference [11]. The latter fact allows them to be used in any city where there is a high concentration of electrical signals $[12,13]$. Their design is often really very simple. The magnetic antenna is made in the form of a closed coil, frame or coil (Fig.1). The simplest magnetic antenna consists of: a core; inductor; coil frame. A frame is provided on the core, and an inductor is wound on the frame. The core of such an antenna is made of magnetic material. Most often made of ferrite, which has good magnetic properties. The core can be made of a longitudinal piece of ferromagnetic, but it is more effective to give this material the shape of a frame. In such a design, the magnetic field will also create an electromotive force (EMF), but variable.

The antenna will turn into an inductor, which converts the energy of the EMF into electrical energy (this is the main task of the antenna). The magnitude of the induced EMF in the frame depends on the position of the structure relative to the plane of the field. The

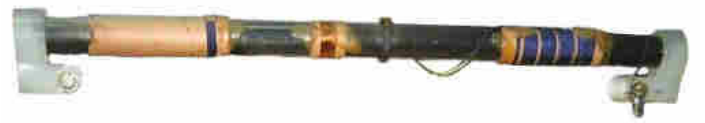

Fig.1. The magnetic antenna

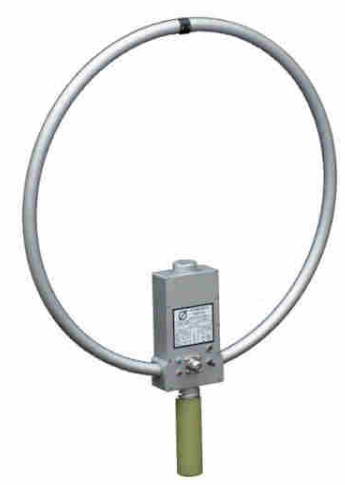

Fig.2. Frame antenna
EMF is maximum if the plane of the turns of the structure is directed to the transmitting station. If you rotate the antenna around the vertical axis (top view), then for one revolution it will have two highs and two lows (zero values) EMF.

EMF in it is given in accordance with the law of electromagnetic induction of M. Faraday [18]:

$$
\varepsilon \lambda=-N \frac{\partial \Phi}{\partial t},
$$

where $N$ - the number of turns,

$\Phi$ - the magnetic flux that permeates the turns and is equal, in turn,

$$
\Phi=\mu \mu_{0} H S
$$

where $H$ - the magnetic field strength of the wave;

$S$ - cross-sectional area of the coil, frame or coil;

$\mu$ - current (effective) magnetic permeability of the core. For ferrite antennas $\mu$ is determined by the size and magnetic permeability of the core. Its approximate values for widespread ferrite rods are in the range from 160 (ferrite $1000 \mathrm{HH}$ ) to 130 (ferrite $400 \mathrm{HH}$ );

$\mu_{0}-$ magnetic permeability of vacuum $\left(\mu_{0}=4 \pi \cdot 10^{-7} \mathrm{H} / \mathrm{m}\right)$.

Just like an electric, a magnetic antenna is characterized by an active height. Given the relationship between $\mathrm{E}$ and $\mathrm{H}$, we can deduce [19]:

$$
h_{d}=\frac{2 \pi \mu S N}{\lambda} .
$$

Now the EMF developed by the antenna is determined by the formula:

$$
\varepsilon=\mathrm{E} \cdot h_{d} .
$$

Magnetic antennas also include frame-type antennas. In addition to the Hertz electric dipole, there is a Hertz magnetic dipole, sometimes called a "frame antenna". The basis of the design of such antennas is a loop of cable 
connected to a capacitor of variable capacity [20]. The perimeter of the loop is usually in the range from to and has the shape of a circle. A circle-shaped antenna is considered a classic loop antenna. Such an antenna can work with many frequency bands, including with shortwave (HF) - band.

This range is interesting because the waves of this range are still propagating in the water. The larger the area of the loop (better if it is round), the greater the signal coverage. Also in the circuit sometimes include a matching transformer. But a frame antenna with a frame perimeter less than 0.1 wavelength is called a "magnetic loop". To further reduce its sensitivity to the electrical component and make a minimum of the pattern deeper and sharper, the frame itself is also shielded (Fig.2).
The pattern of such an antenna will have the shape of infinity or figure eight (Fig.3).

Radio communication on the so-called EHantennas [2] is a new unknown direction. This radio communication was born by the efforts of radio amateurs relatively recently (decades ago), but it seems promising, in particular, because this radio communication can work underwater. The prospect of research (both theoretical and experimental) work in this area is quite broad, as the principle of operation and theoretical foundations of communication on EH-antennas have not yet been established [8]. The reason is that EH-antennas operate on a new, virtually unknown electromagnetic principle, using a previously unknown type of electromagnetic radiation [15 - 16]. The design of EH-antennas consists of two cylinders

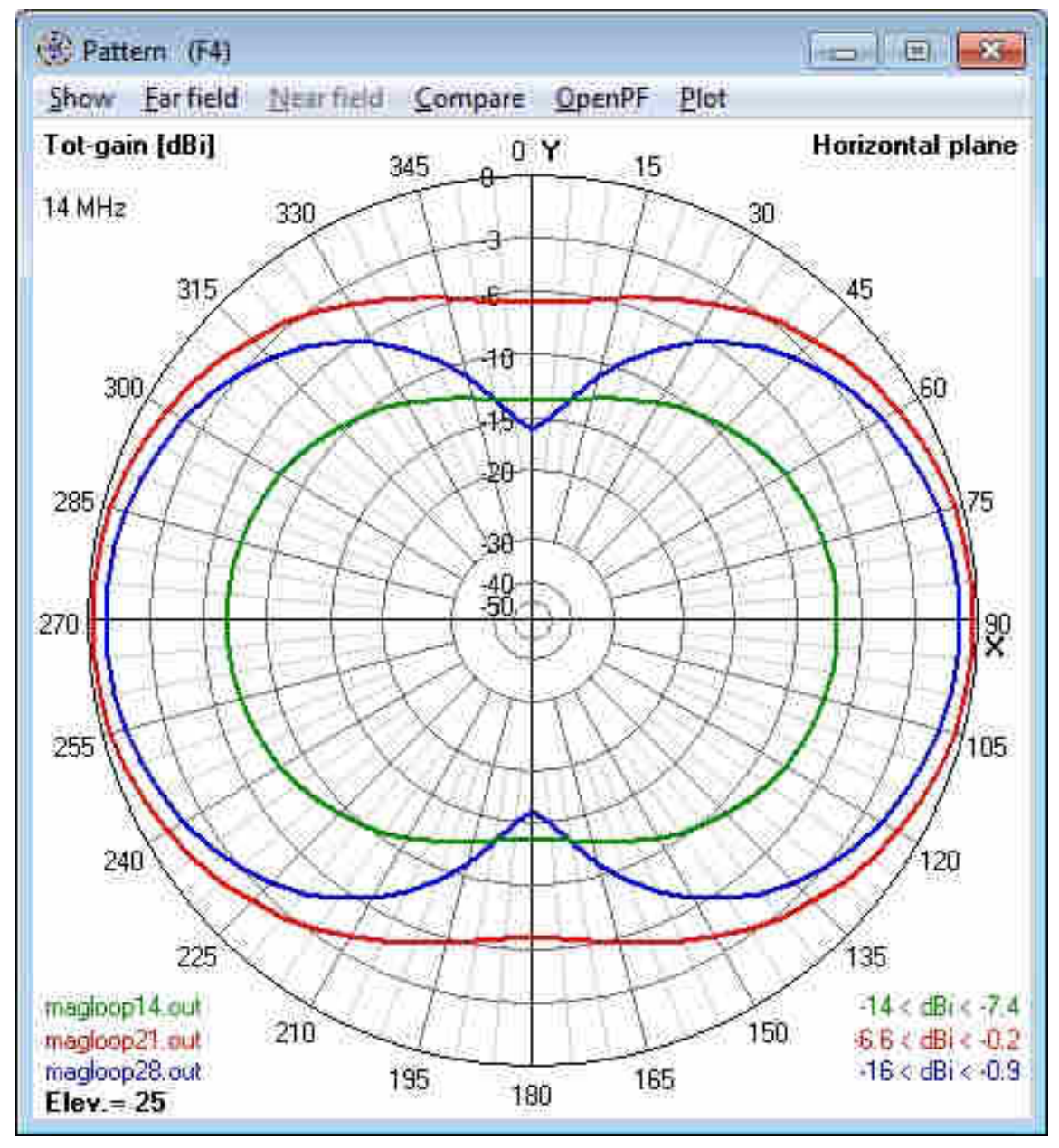

Fig.3. Azimuth Radiation Patterns for Magnetic Loop Antenna modeled for 14, 21 and $28 \mathrm{MHz}$ at 5 feet above Average Ground [14]. As the radiation frequency increases, the antenna efficiency (Fig.4) and the gain (Fig.5) increase, but, unfortunately, the resistance of the aqueous medium increases 


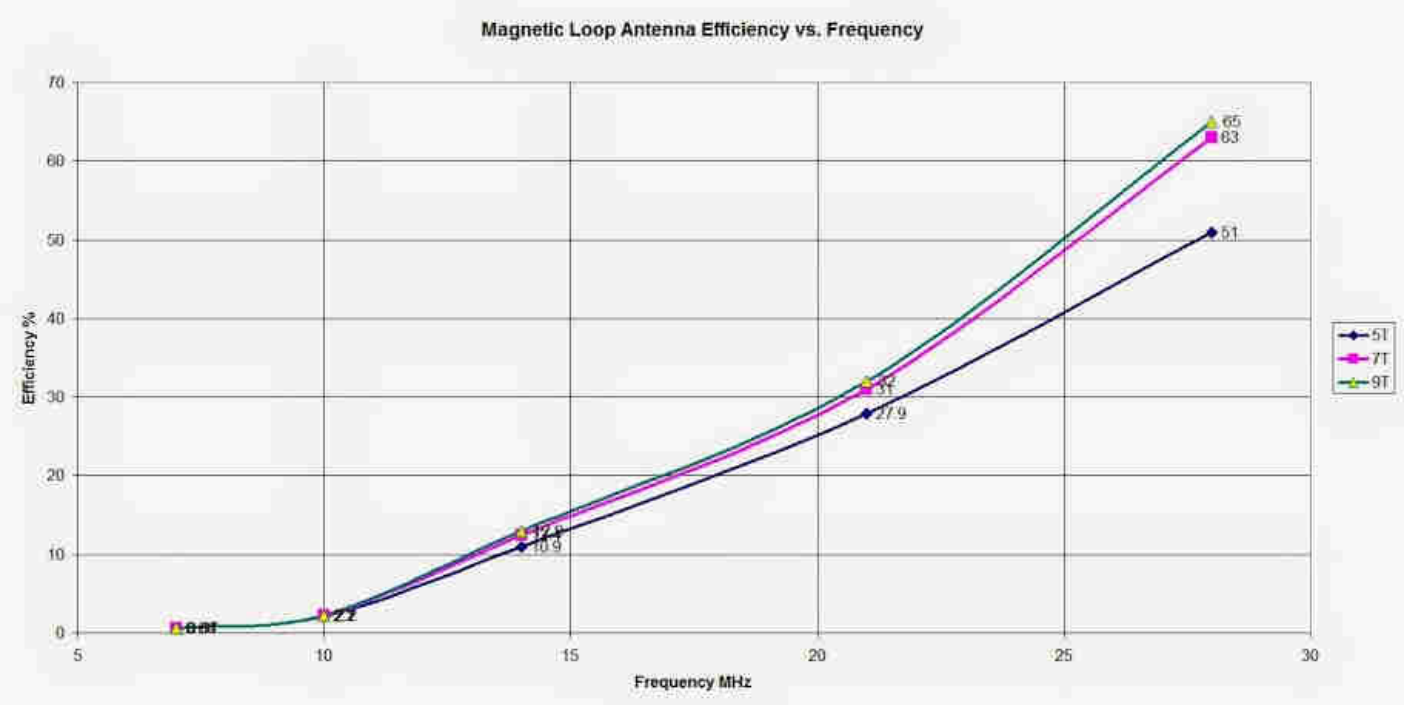

Fig4. Plot of calculated Magnetic Loop Antenna Free Space Efficiency vs. Frequency

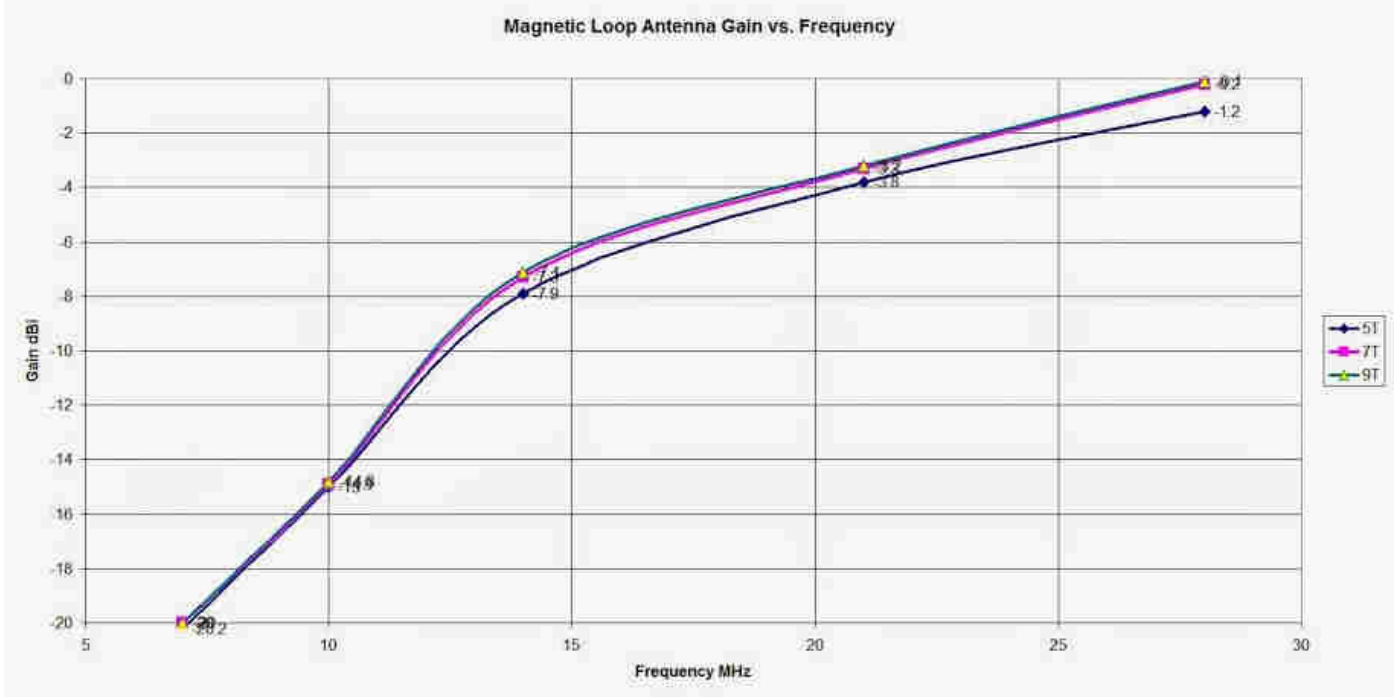

Fig.5. Plot of calculatated Magnetic Loop Antenna Free Space Gain vs. Frequenc

made of non-magnetic material and is made in such a way that the electric charges in its cylinder have a dominant rotational (spin) motion. High voltage is created by a resonant tuning coil, which ends with cylinders (Fig.6). This creates an intense field $\mathrm{E}$ between the cylinders. And this, in turn, creates a very large voltage between the ends of each cylinder, which creates a linear movement of charge on the surface of the cylinders, as in a conventional antenna. These streams allow the EH-antenna to generate the same type of radiation as any conventional Hertz antenna, even though the antenna is very small compared to the wavelength (less than $2 \%$ of the wavelength) [17].

The differential voltage across each cylinder creates a large current and, as a result, a magnetic field. The differential voltage on each cylinder is high, the resistance of the cylinders is low, and therefore the current is large and creates a large magnetic field, despite the small size of the antenna. The ratio of the amplitude between the fields $\mathrm{E}$ and $\mathrm{H}$ is set automatically, forming a very high resistance to radiation. Here is the fundamental difference 
between EH-antennas and all conventional antennas. Thus, conventional antennas operate on the translational motion of electric charges, and the EH-antenna is assumed to operate on the rotational (spin) motion of electric charges. In the radio communication line, the EHantenna works much better with the EHantenna than with the usual one.

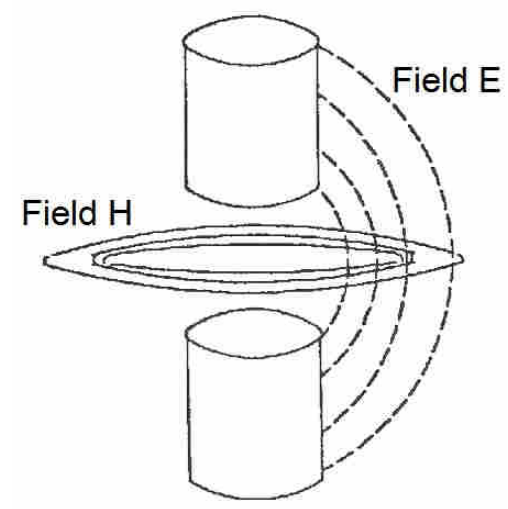

Fig.6. EH-frame antenna

In the study [2] it was determined that the motion of dynamic electrons can have two components - translational and rotational. If so, the resulting electromagnetic field from the motion of these dynamic electrons consists of two complex components, i.e. two separate and distinct electromagnetic fields. The properties of these two electromagnetic fields are very different from each other. In ordinary Hertz antennas there is a translational motion of electrons that form a known electromagnetic field. The peculiarity of En-antennas is that in the cylinders there is also a translational (linear) motion of electrons, but the rotational motions of electrons are dominant.

This situation is created by magnetic fluxes in the tuning coils of the EH-antennas. The magnetic field from the tuning coil penetrates through the cylinders in antiphase to the field caused by currents in the antenna from the phasing coil. Six equations are the result of the application of Maxwell's main two equations, which determine electric and magnetic fields, and supplemented with the assumption of rotation, their application in three dimensions, in comparison with the rectilinear motion of electrons used by Maxwell:

$$
\begin{gathered}
\dot{H}_{x}=\frac{\mathrm{\gamma}^{\prime} \lambda_{\mathrm{x}}}{\pi} \cdot \dot{H}_{0} \cdot \sin \frac{\pi x}{\lambda_{\mathrm{x}}} \cdot \exp \left(i \omega t-\mathrm{\gamma}^{\prime} z\right) ; \\
\dot{H}_{y}=0 ; \\
\dot{H}_{z}=\dot{H}_{0} \cdot \cos \frac{\pi x}{\lambda_{\mathrm{x}}} \cdot \exp \left(i \omega t-\mathrm{\gamma}^{\prime} z\right) ; \\
\dot{E}_{x}=0 ; \\
\dot{E}_{y}=-\frac{i \omega \mu \mu_{0} \lambda_{\mathrm{x}}}{\pi} \cdot \dot{H}_{0} \cdot \sin \frac{\pi x}{\lambda_{\mathrm{x}}} \cdot \exp \left(i \omega t-\mathrm{\gamma}^{\prime} z\right) ; \\
\dot{E}_{z}=0,
\end{gathered}
$$

From these equations we see that even in the simplest wave representation there are two orthogonal magnetic vectors, one of which describes Hertz waves and the other the others. In the general case, it can be represented as

$$
\gamma=\alpha+i \beta
$$

where $\alpha$ characterizes the wave attenuation along the $\mathrm{Oz}$ axis and can be is called the damping coefficient;

$\beta$ characterizes the phase change along axis $\mathrm{Oz}$ and can be called the phase coefficient. From the relation

$$
\left(\frac{m \pi}{\lambda_{\mathrm{x}}}\right)^{2}+\left(\frac{n \pi}{\lambda_{\mathrm{y}}}\right)^{2}=\omega^{2} \mu \mu_{0} \varepsilon \varepsilon_{0}+\left(\mathrm{\gamma}^{\prime}\right)^{2}
$$

we obtain for the region $\lambda_{x}, \lambda_{y}$ :

$$
\left(\mathrm{\gamma}^{\prime}\right)^{2}=\left(\frac{m \pi}{\lambda_{\mathrm{x}}}\right)^{2}+\left(\frac{n \pi}{\lambda_{\mathrm{y}}}\right)^{2}-\omega^{2} \mu \mu_{0} \varepsilon \varepsilon_{0} .
$$

For

$$
\left(\frac{m \pi}{\lambda_{\mathrm{x}}}\right)^{2}+\left(\frac{n \pi}{\lambda_{\mathrm{y}}}\right)^{2}>\omega^{2} \mu \mu_{0} \varepsilon \varepsilon_{0}
$$

we obtain $\left(\gamma^{\prime}\right)^{2}>0$ and $\gamma^{\prime}-$ is a real number, i.e. $\gamma^{\prime}=\alpha$ and $\beta=0$. This case corresponds to a damped wave. 
For

$$
\left(\frac{\mu \pi}{\lambda_{\xi}}\right)^{2}+\left(\frac{v \pi}{\lambda_{\psi}}\right)^{2}<\omega^{2} \mu \mu_{0} \varepsilon \varepsilon_{0}
$$

we obtain $\left(\gamma^{\prime}\right)^{2}<0$ and $\gamma^{\prime}-$ is a imaginary number, i.e. $\gamma^{\prime}=i \beta$ and $\alpha=0$. In this case, we obtain a wave propagating along the axis

$\mathrm{Oz}$ without attenuation. Wherein in the region $\lambda_{x}, \lambda_{y}$, created by the electron (masscharge) corresponds to the critical frequency, determined from the condition $Y^{\prime}=0$ expression:

$$
\omega_{0}=\frac{\pi}{\sqrt{\mu \mu_{0} \varepsilon \varepsilon_{0}}} \cdot \sqrt{\left(\frac{m}{\lambda_{\mathrm{x}}}\right)^{2}+\left(\frac{n}{\lambda_{\mathrm{y}}}\right)^{2}} .
$$

At frequencies below $\omega_{0}$ the waves are damped. At frequencies above $\omega_{0}$ the waves are undamped.

The distance over which an electromagnetic wave propagates during one period of its change, as you know, is called the wavelength

$$
\lambda=v T,
$$

Where

$$
v=\frac{1}{\sqrt{\mu \mu_{0} \varepsilon \varepsilon_{0}}}, T=\frac{2 \pi}{\omega} .
$$

Therefore, the critical frequency $\omega_{0}$ corresponds to the critical wavelength $\lambda_{0}$ in space:

$$
\lambda_{0}=\frac{2 \pi}{\omega_{0} \sqrt{\mu \mu_{0} \varepsilon \varepsilon_{0}}}=\frac{2}{\sqrt{\left(\frac{m}{\lambda_{\mathrm{x}}}\right)^{2}+\left(\frac{n}{\lambda_{\mathrm{y}}}\right)^{2}}}
$$

The quantities $\omega_{0}$ and $\lambda_{0}$ are interrelated with the numbers $m$ and $n$, which determine the nature of the wave. In our case $\lambda_{x}>\lambda_{y}$, therefore the smallest critical frequency is obtained for $m=1$ and $n=0$. It turns out to be equal to:

$$
\omega_{0}=\frac{1}{\sqrt{\mu \mu_{0} \varepsilon \varepsilon_{0}}} \cdot \frac{\pi}{\lambda_{x}}
$$

and, therefore, the longest critical wavelength is $\lambda_{0}=2 \lambda_{x}$. Since for $\omega>\omega_{0}$ we have $\mathrm{V}^{\prime}=i \beta$ and

$$
e^{i \omega t-\gamma^{\prime} z}=e^{i \omega t-\beta z}
$$

to obtain expressions for real instantaneous values of $\dot{H}_{x}, \dot{H}_{y}, \dot{H}_{z}, \dot{E}_{x}$ and $\dot{E}_{y}$ it is necessary in expressions for their complexes, replace the factor $e^{i \omega t-\gamma^{\prime} z}$ with $\sin (\omega t-\beta z)$.

The quantity

$$
\frac{\omega}{\beta}=v^{\prime}
$$

is the phase velocity of the wave.

The wavelength $\Lambda$ along the $\mathrm{Oz}$ axis is obtained from the relation

$$
\beta \Lambda=2 \pi .
$$

Replacing in the relation (1) $\left(\mathrm{Y}^{\prime}\right)^{2}$ by $\omega^{2} \mu \mu_{0} \varepsilon \varepsilon_{0}$ through $\left(\frac{2 \pi}{\lambda}\right)^{2}$ we find:

$$
\beta^{2}=\left(\frac{2 \pi}{\lambda}\right)^{2}-\left(\frac{m \pi}{\lambda_{x}}\right)^{2}-\left(\frac{n \pi}{\lambda_{y}}\right)^{2} .
$$

Then we have

$$
\frac{2}{\Lambda}=\sqrt{\left(\frac{2}{\lambda}\right)^{2}-\left(\frac{m}{\lambda_{\mathrm{x}}}\right)^{2}-\left(\frac{n}{\lambda_{\mathrm{y}}}\right)^{2}}
$$

or

$$
\frac{1}{\Lambda}=\sqrt{\left(\frac{1}{\lambda}\right)^{2}-\left(\frac{1}{\lambda_{0}}\right)^{2}}
$$


This shows that the wavelength $\Lambda$ along the $\mathrm{Oz}$ axis is greater than the wavelength $\lambda$ in the Oxy plane at the same frequency. This difference is the greater, the more $\lambda$ approaches the critical wavelength $\lambda_{0}$, and for $\lambda=\lambda_{0}$ we get $\Lambda=\infty$. Phase velocity can be represented as:

$$
v^{\prime}=\frac{\omega}{\beta}=\frac{\omega}{2 \pi} \cdot \Lambda=\sqrt{\frac{1}{\mu \mu_{0} \varepsilon \varepsilon_{0}}} \cdot \frac{\Lambda}{\lambda}=v \cdot \frac{\Lambda}{\lambda} .
$$

Therefore, the phase velocity $v^{\prime}$ of the electromagnetic waves along the $\mathrm{Oz}$ axis is greater than the speed of electromagnetic waves in the Oxy plane. This, of course, does not mean that the electromagnetic field changes with a speed of 10 greater than $v$, since $v^{\prime}$ is the speed with which the steady-state phase distribution along the $\mathrm{Oz}$ axis changes. In the physical sense this means, that for any violation of the existing mass distribution of the charge the coefficients $m$ and $n$ change, which change the phase distribution along the $\mathrm{Oz}$ axis with a speed $v^{\prime}$. In other words, with this speed, the coefficients $m$ and $n$ change, which determine the frequency filling of the mass-charge wave, i.e. spectrum, which can also be represented by the trigonometric Fourier series whose member numbers correspond to the coefficients $m$ and $n$.

In this case, along the $\mathrm{Oz}$ axis there is a non-zero component of only the magnetic field strength. In this connection, it is of interest that

$$
\nabla B=0
$$

always (the absence of magnetic charges, and the lines of the magnetic field are closed) and magnetic induction is a complex quantity. The magnetic component along the $\mathrm{Oz}$ axis of the critical electron wave (mass charge) is closed at infinity. According to this magnetic wave, the electron (mass-charge) considered in a particular place can be controlled instantly everywhere in the Universe. He constitutes with her one whole, infinitely closed space, where any changes in the phase distribution of his waves are realized instantly in the entire Universe.
The considered properties of the electron component $\dot{H}_{z}$ (mass-charge) fully correspond to the same component of our Earth (mass-charge). It is tempting to influence information on a component $\dot{H}_{z}$ near the Earth. This is the instant passage of information on the universe! Of course, the usual techniques used in conventional radio communications are not applicable here.

The obtained complex expressions of the instantaneous values of the components of the strengths of the magnetic and electric fields $\dot{H}_{x}, \dot{H}_{y}, \dot{H}_{z}, \dot{E}_{x}, \dot{E}_{y}$ and $\dot{E}_{z}$ electron (masscharge) are fully applicable to electrons in the shell of an atom. It also follows from the foregoing that the orbit of an electron (mass charge) in an atom is an epicycloid, and not a distorted circle-ellipse. These complex expressions already in finished form are a kind of periodic wave function of the electron shell of the hydrogen atom $H_{1}$. The region $\lambda_{x}, \lambda_{y}$ determines the external dimensions of the hydrogen atom $H_{1}$. The periodic wave function for electrons in an atom may turn out to be more convenient than transformations of the Schrödinger equation, from which the probability of an electron being at a given point in time at a given point in the orbit is obtained.

EH-antennas are already sufficiently tested in the world and are produced almost industrially [7]. The design of the antenna for this principle is almost patented [10].

A special case of EH-antennas are the socalled HZ-antennas. The device of the HZantenna is quite simple (Fig.7). The antenna consists of two coils arranged coaxially at

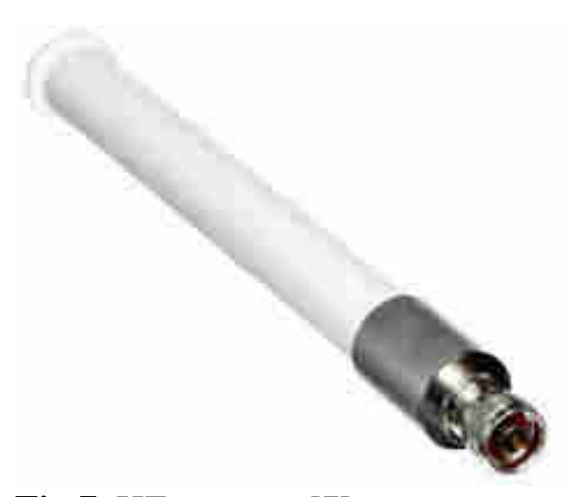

Fig.7. HZ-antenna [7] 
some distance from each other. The coils are included so that their magnetic fields are directed opposite to each other, in other words, there is an anti-phase inclusion of these inductors. To increase the sensitivity, the capacitor is included, which together with the inductors forms a circuit.

This circuit is tuned to the frequency of the received signal. The coils are placed in a copper cylinder or in a copper screen. The magnetic fields of the frame are directed in opposite directions. The field in the far radiation zone, when the distance from the antenna significantly exceeds the diameter of the loop antennas and the distance between them, can be defined as the sum of the fields from each of the loop antennas. This type of antenna is called $\mathrm{Hz}$ antennas and belongs to the class of EH-antennas. Particular attention is drawn to the fact of shielding the antenna. For conventional antennas, such a screen does not allow the antenna to receive a signal from the surrounding space or emit it into space. However, in this case, as studies have shown, the screen not only does not suppress, but also improves the performance of the antenna. Between the same coils, included in antiphase, there is a space of some thickness in which the electric and magnetic fields are zero. The wires that are connected to the coil are also placed in the screen and there is no radiation from them.

Another feature of the HZ-antenna, which was predicted by theory and confirmed in practice, is the high penetrating power of the vector [2]. The field emitted by the HZantenna can penetrate water. This was experimentally established when a small transmitter and an EH antenna enclosed in a plastic sealed housing were placed under water. Both conventional antenna and EH-antenna were used as receiving antennas. Only the EH-antenna was able to receive a signal from a transmitter located under water.

\section{DISCUSSION OF THE RESULTS OF THE STUDY}

Thus, there is a theoretical possibility of organizing underwater radio communication on the principle of using only one component of the electromagnetic wave (magnetic). The use of such radio communication will allow to build communication systems for almost unlimited distance. Issues of energy supply of such systems are subject to further study.

In 2018, a message appeared on the Internet [5] that the Russian Federation has developed technology for the transmission of information and voice data in sea and fresh water, as well as across the border of the environment (water-air). The device provides uninterrupted communication between underwater and surface subscribers. The solution is based on the magnetic component. According to Fig.8, this is a frame magnetic antenna of the HF band, which is described above.

In order to test and develop the theory, it is necessary to conduct further theoretical and experimental research in the following areas: it is necessary to make sure that we are not dealing with an instantaneous, but with a wave process; it is necessary to determine the speed of propagation of the emitted wave. This speed can differ significantly from the speed of light in vacuum and does not depend on $\mu$ and $\varepsilon$, but on other parameters of the medium. This is qualitatively evidenced by experiments on the good passage of a new type of waves through water and reinforced concrete buildings in comparison with electromagnetic waves; it is necessary to study the polarization of these waves. Personal experience (especially the pattern) identifies the moments that indicate the longitudinal nature of these waves; it is also necessary to study the phenomena of reflection from different media, interference of these waves and diffraction.

\section{CONCLUSIONS}

Thus, the following conclusions are possible from the above:

- underwater radio communication can be organized on the principle of separation of the magnetic component of the electromagnetic wave;

- the release of the magnetic component of the electromagnetic wave is carried out in the so-called magnetic antennas; 


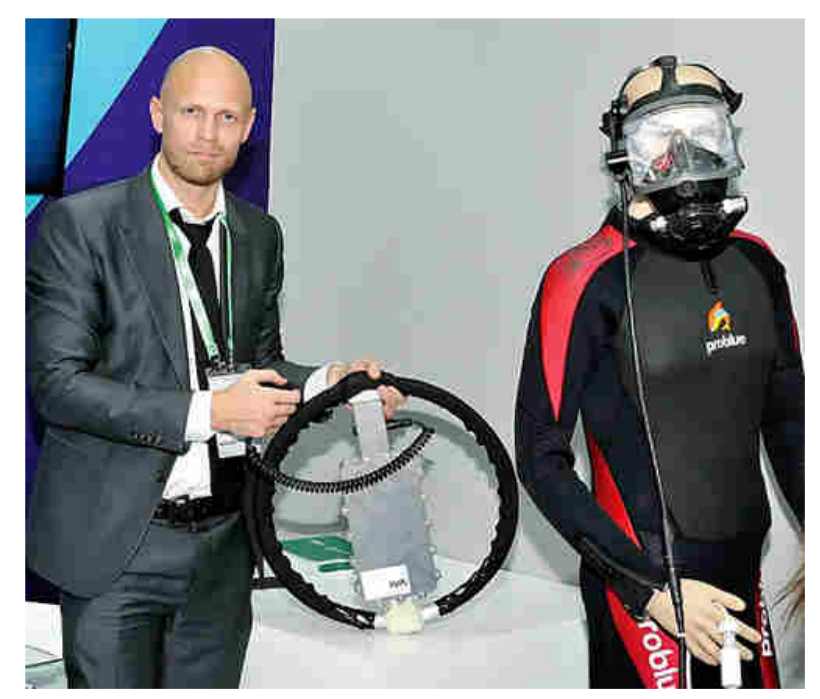

Fig.8. Mobile radio complex of wireless underwater communication IVA S/W

- the magnetic antenna can be built frame, for example [5], or according to the patent [10];

- for the organization of underwater communication, only the antenna-feeder path is subject to change, and other components of the communication channel remain unchanged.

\section{REFERENCES}

1. Dolbnya, 2006. The history of the development of communications with submarines. Marine collection, No 5 (RUS).

2. V.I. Korobeinikov, 2020. The truth and fiction of EH antennas. Received from: https://www.qrz.ru/articles/article282.html (accessed date: 07.07.2020) (RUS).

3. Korochentsev V.I., Rublev V.P., Chayko A.V., 2003. Experimental studies of underground radio communications. Transactions of DVGTU, Iss.133, 5-8 (RUS).

4. Kulakov V.G., 2020. The problem of the radiation power of a magnetic antenna. Received from: http://newidea.kulichki.net/pubfiles/200423182 640.pdf (accessed: 04/23/2020) (RUS).

5. Mobile radio complex wireless underwater communication IVA S/W, 2020. Received from: https://www.iva-tech.ru) (accessed June 7, 2020) (RUS).

6. Radio communication with submarines, 2015. Received from: https://argut.net/blog/ news-radiosvjaz-s-podvodnymi-lodkami.html (accessed October 16, 2015) (RUS).

7. Cisco AIR-ANT2547V-N-HZ= Antenna - 7 dBi, 2020. Received from: https://www. amazon.co.uk/Cisco-AIR-ANT2547V-N-HZAntenna-Outdoor-Wireless/dp/B00X9ZL6C4 (date of the application 07.06.2020).

8. Ted Hart, 2003. EH antennas. ANTENTOP, No 2.

9. V.M. Simulik and I.Yu. Krivsky, 2011. Bosonic symmetries of the Dirac equation. Phys. Lett. A., Vol.375, No 25, 2479-2483.

10. Robert T. Hart, Vladymir I. Korbihikov, 2007. ANTENNA FOR ELECTRON SPIN RADATION. Patent United States No.: US 2007/0013595 A1 US 20070013595A1, Pub. Date: Jan. 18. 2007.

11. B. Bhattacharya and S. Mondal, 2013. Probability of Reception of Jovian Bursts as Derived from Io-Phase and the Location of Central Meridian Longitude. IJECT, Vol.04, 104-106.

12. B. Ismail and M. K. Hisham, 2010. Listening to Jupiter's signal using Radio Telescope Recorder, ICCDA, Vol.01, 278-282.

13. B. Bhattacharya et al., 2012. Detection Of Jovian Radio Bursts At High Altitudes. IJEST, Vol.04, No 06, 3029-3038.

14. J. L. Lombardero, 2010. Radiotelescopio loop. CPAN-Ingenio, 1-16. Received from: https://www.i-cpan.es/concurso4/docs/ radiotelescopio-loop.pdf.

15. W. Kwon et al., 2013. A magnetic resonant loop antenna to enhance the operating distance of $13.56 \mathrm{MHz}$ RFID systems. ISOCC IEEE, 013-014.

16. F. El Hatmi et al., 2011. Magnetic loop antenna for wireless capsule endoscopy inside the human body operating at $315 \mathrm{MHz}$ : Near field behavior. MMS IEEE, 81-87.

17. H. Martinez and M. R Ghezzi, 2014. La antenna cuadro o Magnetic loop. SOLVEGJ Comunicaciones, 1-13. Received from: http://lu6etj.hostargentina.com.ar/lu6etj/tecnico s/loop/antena_de_cuadro.htm.

18. N. K. Nikolova, 2014. Hamilton, Loop Antennas. Received from: http://www.antentop. org/004/files/tr004.pdf.

19. S. Yates, 2013. Small Transmitting Loop Antennas: Magnetic Loop Antennas. Texas, USA. Received from: http://www.aa5tb.com/loop. html.

20. H. Rodrigues, 2015. Development of a Magnetic Loop Antenna for the Detection of Jovian Radiowaves at $20.1 \mathrm{MHz}$. Received from: 
http://www.scielo.org.co/pdf/tecci/v11n20/v11n 20a06.pdf.

21. Bobrovsky I. V., 2013. Experimental studies of the acoustic communication system in shallow water. Acoustic magazine, No 59, Vol.06, 667676.

22. Kirill Otradnov, Volodymyr Shuliak, Sergii Kornieiev, 2017. Underwater wireless video communication in operations of AUV/UUVs - new horizon of underwater explorations. Underwater Technologies, Vol.06, 46-56.

\section{Устройство подводной радиосвязи}

\section{Юрий Хлапонин, Александр Селюков}

Аннотация. Создание устройства радиосвязи для подводных объектов для обмена голосовыми сообщениями и для передачи цифровых данных. Актуальность этой проблемы определена тем, что существующие на сегодня образцы оборудования подводного радиосвязи является чрезмерно мощными, габаритными и с низкою пропускной способностью. Анализ разработок в этой области показал, что подводную радиосвязь можно организовать на продольных электромагнитных волнах или с помощью использования только магнитной составляющей электромагнитной волны.

Принцип работы устройства основан на использовании только магнитной составляющей электромагнитной волны. Известно, что электромагнитная волна состоит из двух составляющих: электрической и магнитной. Вода - это электропроводящая среда, где действует скинэффект, по которому чем глубже, тем сигнал слабее. Но это для электрической составляющей. Вода имеет слабые магнитные свойства, поэтому для магнитной составляющей электромагнитной волны вода - не помеха.
Такое устройство в зависимости от его мощности может быть использовано как для индивидуальной связи пловцов, так и для организации радиосвязи между радиобуями и даже для связи с подводными лодками. Выделить магнитную составляющую электромагнитной волны возможно за двумя принципами: усилить магнитную составляющую или ослабить электрическую составляющую.

Выделение магнитной составляющей электромагнитной волны осуществляется в так называемых магнитных антеннах, которые могут быть построены в виде катушек с ферритовым сердечником, могут быть рамочного типа определенного размера или в виде катушки в экране из немагнитного металла.

При модернизации устройств обычной радиосвязи в подводный вариант их использования изменениям подлежит только антеннофидерный тракт, а другие составляющие канала связи остаются без изменений. Применение таких принципов организации радиосвязи позволит строить системы подводной связи для практически неограниченного расстояния.

Ключевые слова: радиосвязь, антенна, электромагнитная волна, электромагнитное поле, магнитная составляющая, электродвижущая сила, подводная лодка. 